\title{
AN ANALYTICAL TECHNIQUE FOR THE SEISMIC RESPONSE ASSESSMENT OF SLENDER BRIDGE PIERS
}

\author{
E. Tubaldi ${ }^{1}$, L. Tassotti ${ }^{2}$, A. Dall'Asta ${ }^{1}$, L. Dezi ${ }^{2}$ \\ ${ }^{1}$ School of Architecture and Design (SAD), University of Camerino \\ Viale della Rimembranza, 63100, Ascoli Piceno (AP), Italy \\ e-mail: etubaldi@gmail.com, andrea.dallasta@unicam.it \\ ${ }^{2}$ Department of Civil Engineering, Construction and Architecture (DICEA), Polytechnic University of \\ Marche, Via Brecce Bianche, 60131 Ancona (AN), Italy \\ e-mail: tassottiluca@gmail.com,1.dezi@univpm.it
}

Keywords: Axial load effects, Analytical model, Vibration, Earthquake Engineering.

Abstract. This work proposes an analytical technique for the analysis of the effects of axial loads on the dynamic behaviour and seismic response of tall and slender bridge piers. The pier is modeled as a linear elastic Euler-Bernoulli cantilever beam with uniformly distributed mass, representing the pier mass, and a tip mass at the free end, representing the deck mass. The beam is subjected to the reaction due to deck loads, and to the pier self weight.

The seismic problem is described by a linear partial differential equation of seismic motion with variable coefficients. This equation is decoupled by using the exact vibration modes which are obtained by applying the Frobenius method. By this way, the seismic response can be evaluated in terms of superposition of modal contributions.

The proposed formulation is applied to the seismic analysis of a realistic slender bridge pier. The results of the study permit to shed light on the effect of axial load on the various vibration modes that contribute to the seismic response and to evaluate the suitability of amplification factors commonly used by seismic codes to account for axial load effects. 


\section{INTRODUCTION}

A large number of bridges over mountain areas and deep valleys are built on tall piers. Because of the rugged topography of these areas, the height of bridge piers could even reach 200 $\mathrm{m}$. The seismic assessment and design of slender piers usually differs from that of short piers because of the influence of axial load effects on the dynamic behaviour.

It is well known that axial load effects induce in general a reduction of the transverse bending stiffness and this may have different consequences on the transverse structural response depending on the type of loading experienced by the system. In the case of static loadings, the axial load effects usually increase the response beyond the values obtained by first order analysis. On the other hand, in the case of dynamic loadings such as earthquake-induced loadings, the interaction between axial loads and transverse displacements induces changes in the system dynamic properties and elastic periods elongation $[1,2]$, which in turn may result on either a decrease or an increase of displacements and internal action demands.

In bridge engineering-design practice, the axial-load effects are usually taken into account in a simplified manner by introducing an amplification factor for the piers seismic moments [3] evaluated via first-order analysis. Many studies in past years have been devoted to the calibration of expressions for the amplification factor [1,4-9], on the basis of simple singledegree-of-freedom (SDOF) models. These models often consisted in a rigid inverted pendulum with an elasto-plastic rotational restraint at the base, and a tip mass at the free end with a concentrated weight force. Recent works $[10,11]$ extended the application of the amplification factor method also to the direct displacement based design of bridge piers. Other alternative methods were defined to account for axial load effects in a simplified way, without recourse to the amplification factor. In this context, the method proposed in [12] introduced the concepts of effective height and yield point spectra for the design of piers behaving as SDOF system.

The main limitation of the SDOF model employed in these studies is that it is adequate to represent only short bridge piers, for which the inertia is concentrated at the top whereas the distributed pier mass and the relevant variation of axial loads can be neglected. Slender piers, as those considered in this study, are on the contrary characterized by significant distributed masses and the analysis of their dynamic behavior should also consider higher modes effects [13]. Only few studies analyzed the axial-load effects on slender piers by employing refined multi-degree-of- freedom (MDOF) models. In this context are the work of [14] and [15], analyzing the evolution of plastic hinges formation in tall piers, and the works of [16] and [17], focusing more on the nonlinear modeling of the problem.

In this paper, an analytical continuous model is specifically developed to analyze the dynamic and seismic response of slender bridge piers by including axial load effects and higher mode effects as induced by masses distributed along the pier length. The model consists of a linear-elastic Euler-Bernoulli cantilever beam with a concentrated mass at the pier top and a uniform distributed mass, prestressed by a linearly varying axial load representing the deck loads and the pier self weight. The choice of employing a continuous model instead of a discrete model is motivated by the fact that the former permits to derive an exact solution of the dynamic and seismic problem, by making explicit the characteristic parameters that control the behaviour of the system. This is also convenient for performing parametric studies and for developing and validating more efficient and accurate numerical methods. It is noteworthy that the model considered in this paper is linear elastic. Although bridge piers can be designed to yield in order to dissipate the seismic energy [3], the high flexibility of slender piers is such that the displacement demand imposed by the seismic excitation is very unlikely to induce 
inelastic deformations. Therefore, the linear elastic model appears adequate for slender piers under moderate seismic excitations.

In this paper, the equation of perturbed motion in the neighborhood of the axially loaded configuration of the pier is derived by applying the D'Alembert principle. The seismic response is expressed in terms of superposition of the contributions from the exact vibration modes of the system. In order to decouple the equation of motion, the exact orthogonality conditions for the vibration modes are derived. It is noted that the eigenvalue problem that needs to be solved to determine the vibration modes of the systems involves a differential equation with variable coefficients. The solution of this problem is obtained by extending previous formulations and results for similar problems [2, 18-21], in particular by application of the Frobenius method [19].

The proposed formulation and analysis technique are applied to a case study consisting of slender RC piers belonging to a three-span bridge. The followed approach, based on exact mode superposition, permits to evaluate the effect of axial loads separately on each mode contributing to the seismic response.

\section{ANALYTICAL MODEL AND FORMULATION}

The slender pier is modeled (Fig. 1) as an Euler-Bernoulli cantilever beam with stiffness $b(x)$, mass per unit length $m(x)$, and with a tip mass $M$ at the top. In the reference configuration, the beam is subjected to a concentrated compression force, $P$, at the free end, and to a distributed compressive load, $m(x) g$, corresponding to the pier self weight, producing an internal compressive action $N(x)$ expressed as (positive if compressive):

$$
N(x)=P+\int_{x}^{H} m(\xi) g d \xi
$$

The concentrated mass and force at the pier top represent the contribution respectively to loads and inertia of the portion of the deck sustained by the pier. In general, $P$ may differ from $M g$.
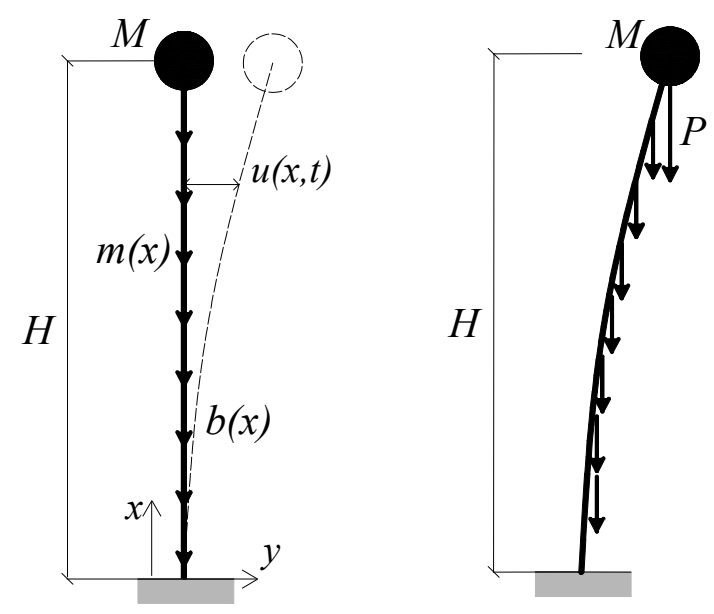

Fig. 1. a) Geometry of the problem and pier model, b) deformed pier configuration.

Let $V=\left\{v(x) \in L^{2}[\Omega]: v(0)=v^{\prime}(0)=0\right\}$ be the space of transverse displacement functions defined along the spatial interval $\Omega=[0, H]$ and satisfying the kinematic (essential) 
boundary conditions, and $u(x, t) \in U \subseteq C^{2}\left(V ;\left[t_{0}, t_{1}\right]\right)$ be the motion (Fig. 1), defined in the time interval considered $\left[t_{0}, t_{1}\right]$. The known functions $b(x), N(x), m(x)$ are continuous, bounded and positive. The differential dynamic problem can be derived from the D'Alembert principle. This variational principle states that the external virtual work $\delta W_{E}(u, \eta)$ resulting from external and inertia forces acting through every virtual displacement $\eta \in V$ consistent with the geometric restrains, is equal to the internal virtual work $\delta W_{I}(u, \eta)$ resulting from the stresses acting through the corresponding virtual strains. The dynamic problem describing the infinitesimal perturbed motion in the neighborhood of the axially-loaded reference configuration has the following expression [22]:

$$
\begin{aligned}
& \int_{0}^{H} b(x) u^{\prime \prime}(x, t) \eta^{\prime \prime}(x) d x-N(x) u^{\prime}(x, t) \eta^{\prime}(x)+m(x) \ddot{u}(x, t) \eta(x) d x+M \ddot{u}(H, t) \eta(H) d x= \\
& =-\left[\int_{0}^{H} m(x) \eta(x) d x-M \eta(H)\right] \ddot{u}_{g}(t) \quad \forall \eta \in V ; \forall t \in\left[t_{0}, t_{1}\right]
\end{aligned}
$$

where $\ddot{u}_{g}(t)$ denotes the ground motion input. The problem is completed by assigning the initial conditions $u\left(x, t_{0}\right)=\dot{u}\left(x, t_{0}\right)=0$. Eqn. (2) can be formally rewritten as:

$$
\mathrm{K}(u, \eta)-\mathrm{N}(u, \eta)+\mathrm{M}(\ddot{u}, \eta)=-\ddot{u}_{g} \mathrm{M}^{*}(\eta) \quad \forall \eta \in V ; \forall t \in\left[t_{0}, t_{1}\right]
$$

where $\mathrm{K}, \mathrm{N}, \mathrm{M}$ are bilinear symmetric forms mapping functions defined in the spatial interval $V$ into real numbers, and $\mathrm{M}^{*}: V \rightarrow \mathbb{R}$ is a linear form. They are defined as follows:

$$
\begin{aligned}
& \mathrm{K}(u, \eta)=\int_{0}^{H} b(x) u^{\prime \prime}(x, t) \eta^{\prime \prime}(x) d x \\
& \mathrm{~N}(u, \eta)=\int_{0}^{H} N(x) u^{\prime}(x, t) \eta^{\prime}(x) d x \\
& \mathrm{M}(\ddot{u}, \eta)=\int_{0}^{H} m(x) \ddot{u}(x, t) \eta(x) d x+M \ddot{u}(H, t) \eta(H) \\
& \mathrm{M}^{*}(\eta)=\int_{0}^{H} m(x) \eta(x) d x+M \eta(H)
\end{aligned}
$$

The strong form of the seismic problem can be derived through integration by parts of the terms $\mathrm{K}, \mathrm{N}, \mathrm{M}$ in Eqn. (2). The following partial differential equation of motion and the relevant boundary conditions is obtained:

$$
\begin{aligned}
& {\left[b(x) u^{\prime \prime}(x, t)\right]^{\prime \prime}+\left[N(x) u^{\prime}(x, t)\right]^{\prime}+m(x) \ddot{u}(x, t)=-m(x) \ddot{u}_{g}(t)} \\
& u(0, t)=u^{\prime}(0, t)=b(H) u^{\prime \prime}(H, t)=0 \\
& b(H) u^{\prime \prime \prime}(H, t)+P u^{\prime}(H, t)-M \ddot{u}(H, t)=M \ddot{u}_{g}(t) \\
& u\left(x, t_{0}\right)=\dot{u}\left(x, t_{0}\right)=0
\end{aligned}
$$




\section{EIGENVALUE PROBLEM}

The free vibrations of the system can be studied by analyzing the homogeneous problem $\left(\ddot{u}_{g}=0\right)$. As usual, the problem is solved by separation of variables technique. The motion $u(x, t)$ is expressed in terms of the product of a spatial function $\psi(x) \in V$ and of a timedependent function $Z(t)=Z_{0} e^{i \omega t}$ as follows:

$$
u(x, t)=\psi(x) Z(t)
$$

After substituting Eqn.(6) into Eqn.(5) for $\ddot{u}_{g}=0$, the following boundary-value problem is obtained:

$$
\begin{aligned}
& {\left[b(x) \psi^{\prime \prime}(x)\right]^{\prime \prime}+\left[N(x) \psi^{\prime}(x)\right]^{\prime}-m(x) \omega^{2} \psi(x)=0} \\
& \psi(0)=\psi^{\prime}(0)=\psi^{\prime \prime}(H)=0 \\
& b(H) \psi^{\prime \prime \prime}(H)+P \psi^{\prime}(H)+\omega^{2} M \psi(H)=0
\end{aligned}
$$

It is noteworthy that Eqn.(7) is satisfied by an infinite set of eigenvalues, $\omega_{s}$, and of eigenvectors, $\psi_{s}(x)$, for $s=1,2, \ldots, N$.

\subsection{Generalized orthogonality conditions for vibration modes}

The orthogonality conditions, useful to decouple the equation of motion, can be obtained from the weak form corresponding to Eqn. (3) and written for the generic $s$-th vibration mode:

$$
\mathrm{K}\left(\psi_{s}, \eta\right)-\mathrm{N}\left(\psi_{s}, \eta\right)-\omega_{s}^{2} \mathrm{M}\left(\psi_{s}, \eta\right)=0 \quad \forall \eta \in V
$$

Substituting $\eta$ by $\psi_{m}$ in Eqn. (8) written for mode $s=l$, and substituting $\eta$ by $\psi_{l}$ in Eqn. (8) written for mode $s=m$, one has:

$$
\begin{aligned}
& \mathrm{K}\left(\psi_{l}, \psi_{m}\right)-\mathrm{N} \quad\left(\psi_{l}, \psi_{m}\right)-\omega_{l}^{2} \mathrm{M}\left(\psi_{l}, \psi_{m}\right)=0 \\
& \mathrm{~K}\left(\psi_{m}, \psi_{l}\right)-\mathrm{N} \quad\left(\psi_{m}, \psi_{l}\right)-\omega_{m}^{2} \mathrm{M}\left(\psi_{m}, \psi_{l}\right)=0
\end{aligned}
$$

After subtracting Eqn. (10) from Eqn. (9), by virtue of the symmetry of the forms $\mathrm{K}, \mathrm{M}, \mathrm{N}$, one obtains the first generalized orthogonality condition:

$$
\left(\omega_{m}^{2}-\omega_{l}^{2}\right) \mathrm{M}\left(\psi_{l}, \psi_{m}\right)=0
$$

Since $\omega_{l} \neq \omega_{m}$, Eqn.(11) also reads as:

$$
\mathrm{M}\left(\psi_{l}, \psi_{m}\right)=M \psi_{l}(H) \psi_{m}(H)+\int_{0}^{H} m(x) \psi_{l}(x) \psi_{m}(x) d x=0
$$

Upon substitution of Eqn. (12) into Eqn. (9), the second orthogonality condition is obtained:

$$
\mathrm{K}\left(\psi_{l}, \psi_{m}\right)-\mathbf{N}\left(\psi_{l}, \psi_{m}\right)=\int_{0}^{H} b(x) \psi_{l}^{\prime \prime}(x) \psi_{m}^{\prime \prime}(x) d x-\int_{0}^{H} N(x) \psi_{l}^{\prime}(x) \psi_{m}^{\prime}(x) d x=0
$$


It is noteworthy that a closed-form analytical expression of the circular frequency for the $s$ th vibration mode can be derived from Eqn. (8) for $\eta=\psi$ s and reads as follows:

$$
\omega_{s}^{2}=\frac{\mathrm{K}\left(\psi_{s}, \psi_{s}\right)-\mathrm{N}\left(\psi_{s}, \psi_{s}\right)}{\mathrm{M}\left(\psi_{s}, \psi_{s}\right)}=\frac{\bar{k}_{s}}{\bar{m}_{s}}
$$

where $\bar{k}_{s}$ and $\bar{m}_{s}$ are the generalized stiffness and mass of the $s$-th mode.

\subsection{Eigenvalue problem solution}

The boundary-value problem corresponding to Eqn.(5) contains a differential equation with variable coefficients. A closed-form solution for this problem can be obtained through the Frobenius method by expressing the eigenvectors in terms of power series [19]. Appendix A reports the application of the Frobenius method to the specific problem analyzed, in the case of uniform distributed mass and stiffness through the pier length. It is noteworthy that the proposed solution can be regarded as "exact" only for an infinite series expansion and that for practical purposes the series is truncated at some order.

\section{SEISMIC RESPONSE BY MODE SUPERPOSITION METHOD}

In this study, the exact mode superposition method is employed to obtain a solution of the seismic problem corresponding to the assessment of the system response under the earthquake input. The advantage of this method over other analysis method is that it permits to represent the motion as the sum of the contributions of the exact vibration modes of the system. This allows to evaluate separately the contribution of each vibration mode to the response parameters of interest, and also to evaluate the effect of axial loads on the modal responses.

The motion is expressed as the following summation series:

$$
u(x, t)=\sum_{s=1}^{\infty} \psi_{s}(x) q_{s}(t)
$$

where $\psi_{s}(x) \in V$ is the $s$-th mode shape, and $q_{s}(t):\left[t_{0}, t_{1}\right] \rightarrow \mathbb{R}$ is the corresponding generalized coordinate.

Substituting Eqn. (15) into Eqn. (3), one obtains:

$$
\sum_{s=0}^{\infty} \ddot{q}_{s}(t) \mathrm{M}\left(\psi_{s}, \eta\right)+q_{s}(t)\left[\mathrm{K} \quad\left(\psi_{s}, \eta\right)-\mathrm{N} \quad\left(\psi_{s}, \eta\right)\right]=-\ddot{u}_{g}(t) \mathrm{M}^{*}(\eta) \quad \forall \eta \in V
$$

Substituting in the both sides of Eqn. (16) $\eta$ by the $m$-th eigenfunction $\psi_{m}(x)$ one obtains:

$$
\sum_{l=0}^{\infty} \ddot{q}_{s}(t) \mathrm{M}\left(\psi_{s}, \psi_{m}\right)+q_{s}(t)\left[\mathrm{K} \quad\left(\psi_{s}, \psi_{m}\right)-\mathrm{N} \quad\left(\psi_{s}, \psi_{m}\right)\right]=-\ddot{u}_{g}(t) \mathrm{M}^{*}\left(\psi_{m}\right) \quad \forall \psi_{m} \in V(17
$$

This is evidently a diagonal problem, by virtue of the two previously derived orthogonality conditions. The $s$-th decoupled equation reads as follows:

$$
\ddot{q}_{s}(t)+\omega_{s}^{2} q_{s}(t)=-\rho_{s} \ddot{u}_{g}(t)
$$

where $\omega_{s}^{2}$ is the circular frequency of Eqn. (14) and $\rho_{s}$ denotes the $s$-th mode participation factor, expressed as: 


$$
\rho_{s}=\frac{\mathrm{M}^{*}\left(\psi_{s}\right)}{\mathrm{M}\left(\psi_{s}, \psi_{s}\right)}
$$

Eqn.(18) represents the equation of motion of a generalized SDOF system with circular vibration frequency $\omega_{s}$, subjected to the seismic input $\rho_{s} \ddot{u}_{g}(t)$. A viscous damping source is added a posteriori to this system, thus resulting in the following equation:

$$
\ddot{q}_{s}(t)+2 \xi_{s} \omega_{s} \dot{q}_{s}(t)+\omega_{s}^{2} q_{s}(t)=-\rho_{s} \ddot{u}_{g}(t)
$$

where $\xi_{s}$ denotes the $s$-th mode damping factor. The values of $\xi_{s}$ for the various vibration modes can be calibrated based on experimental observations [13].

By applying the mode superposition method, Eqn.(15) can be expressed as follows:

$$
u(x, t)=\sum_{s=1}^{\infty} \rho_{s} \psi_{s}(x) D_{s}(t)
$$

where $D_{s}(t)$ denotes the response of an oscillator with natural frequency $\omega_{s}$ subjected to the seismic input $\ddot{u}_{g}(t)$ and homogeneous initial conditions

$$
D_{s}(t)=\frac{1}{\omega_{d s}} \int_{0}^{t} \ddot{u}_{g}(\tau) e^{-\xi_{s} \omega_{s}(t-\tau)} \sin \left[\omega_{d s}(t-\tau)\right] d \tau
$$

Having evaluated the displacement response history, the histories of the bending moment and of the shear along the beam can be obtained as follows:

$$
\begin{gathered}
M(x, t)=-b(x) u^{\prime \prime}(x, t)=-b(x) \sum_{s=1}^{\infty} \rho_{s} \cdot \psi_{s}^{\prime \prime}(x) \cdot D_{s}(t) \\
V(x, t)=\left[-b(x) u^{\prime \prime}(x, t)\right]^{\prime}-N(x) u^{\prime}(x, t)=-\sum_{s=1}^{\infty}\left\{\left[b(x) \psi_{s}^{\prime \prime}(x)\right]^{\prime}+N(x) \psi_{s}^{\prime}(x)\right\} \rho_{s} \cdot D_{s}(t)
\end{gathered}
$$

\section{SEISMIC RESPONSE OF A CONTINUOUS BRIDGE WITH SLENDER PIERS}

\subsection{Case study}

In this section, the application of the proposed analysis technique is illustrated by considering the case study of a realistic bridge slender pier subjected to earthquake excitation. The pier belong to a three-span bridge [24] consisting of a steel-concrete composite deck (with span length of $60 \mathrm{~m}+80 \mathrm{~m}+60 \mathrm{~m}$ ) and of two identical RC piers. These piers, of height $H=40$ $\mathrm{m}$, have a circular hollow transverse section with external diameter of $4.0 \mathrm{~m}$ and internal diameter of $3.2 \mathrm{~m}$. The pier head has a rectangular transverse section with dimensions $4.0 \mathrm{~m} \mathrm{x}$ $8.0 \mathrm{~m}$, and is $1.5 \mathrm{~m}$ high. The longitudinal rebars reinforcement ratio is $1.5 \%$. Class C30/37 concrete is used for concrete and S500 steel is used for the longitudinal rebars [25].

The pier effective stiffness accounting for concrete cracking is $b(x)=E I=7.3086 \cdot 10^{7}$ $\mathrm{kNm}^{2}$, the pier distributed mass is $m(x)=\bar{m}=11.53 \mathrm{ton} / \mathrm{m}$, whereas the mass concentrated at the top and describing the deck and pier head inertia is $M=2145$ ton. The axial force along the pier is a linear function of the form $N(x)=P+\bar{m} g(H-x)$, where $P$ is the weight of the 
deck acting at the top of the pier equal to $15470 \mathrm{kN}$. The value of the buckling load of a cantilever beam with the same flexural stiffness of the pier is $P_{c r}=\pi^{2} E I / 4 H^{2}=1.27 \cdot 10^{8} \mathrm{kN}$, corresponding to a ratio $P / P_{c r}=0.18$.

The seismic action is defined by the EC8 type I soil type B (soil factor $\mathrm{S}=1.20$ ) spectrum [3], for an importance factor $\gamma_{I}=1$ and a peak ground acceleration PGA $=0.30 \mathrm{~g}$. The bridge has been designed for limited ductile behaviour, because the piers are very flexible and there is no need for dissipative behaviour or seismic isolation.

The analytical model and analysis technique proposed in this study are used to investigate the response of one of the two equal piers under a seismic input acting along the longitudinal direction.

a)

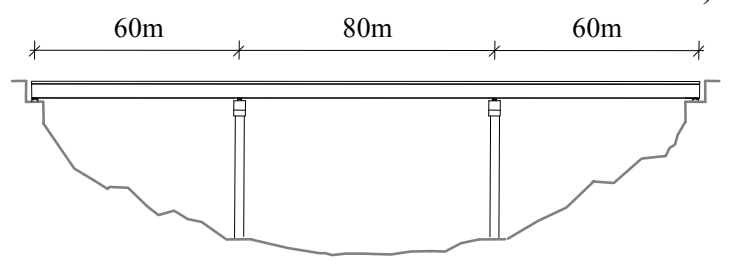

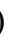

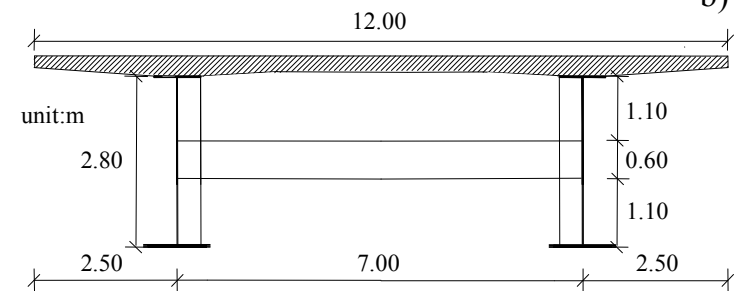

Fig. 2. Bridge geometry: a) longitudinal profile, b) transverse deck cross-section.

\subsection{Modal analysis}

The eigenvalue problem is solved by applying the Frobenius method as described in Appendix A. The first 6 vibration periods and modes of the system have been evaluated by truncating the series expansion of the modal shapes after 100 terms. It is noteworthy that the number of terms required to obtain accurate estimates of the vibration periods increases with increasing mode order. In fact, while the estimate of the fundamental vibration periods exhibits negligible variations for increasing number of terms beyond 5, to estimate accurately the sixth vibration periods 100 terms are needed.

The first three longitudinal vibration periods of the pier in the longitudinal direction disregarding axial load effects are $5.03 \mathrm{~s}, 0.26 \mathrm{~s}$, and $0.08 \mathrm{~s}$, in agreement with the values reported in [24] and based on a bridge refined finite element model. Table 1 reports the modal vibration periods and participation factors obtained by accounting for axial load effects and by disregarding axial load effects (i.e., for $N(x)=0$ ).

\begin{tabular}{c|c|c|c|c|}
\multirow{2}{*}{ Mode } & \multicolumn{2}{|c|}{ Without axial load effects } & \multicolumn{2}{c|}{ With axial load effects } \\
\cline { 2 - 5 } & $T_{i}[\mathrm{~s}]$ & $M_{\mathrm{i}}[\%]$ & $T_{i}[\mathrm{~s}]$ & $M_{\mathrm{i}}[\%]$ \\
\hline 1 & 5.0328 & 91.4915 & 5.4023 & 91.4620 \\
2 & 0.2512 & 4.9658 & 0.2532 & 4.5479 \\
3 & 0.0781 & 1.2494 & 0.0784 & 1.4117 \\
4 & 0.0375 & 0.7667 & 0.0376 & 0.6769 \\
5 & 0.0220 & 0.3454 & 0.0220 & 0.3975 \\
6 & 0.0144 & 0.2966 & 0.0144 & 0.2592
\end{tabular}

Table 1. Modal analysis results accounting for and disregarding the second-order effects.

It is observed that only the first mode vibration period is significantly affected by the axial load effects. An opposite trend can be observed for the mass participating factors where variations increase for higher order modes. 
Fig. 3 reports the first two modal shapes obtained accounting for and disregarding the effect of axial loads. The modal shapes are normalized with respect to the norm $\left\|\psi_{l}\right\|_{2}=\left(\int_{0}^{H} \psi_{l}(x)^{2} d x\right)^{1 / 2}$. With reference to the first mode, the influence of axial load increases for increasing derivative order. In fact, the displacement shape is practically the same as that obtained by neglecting axial load effects, whereas the second order (proportional to the bending moment) and particularly the third order derivative (related to the shear) are significantly affected by axial load effects. The influence of axial load effects on the second and higher vibration modes and relevant derivatives is practically negligible.
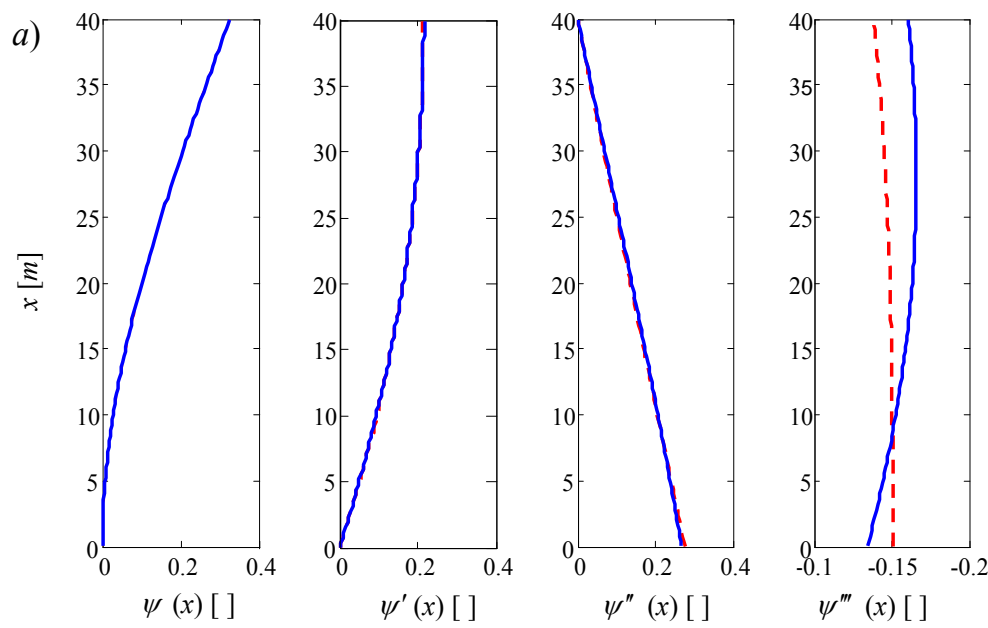

W second-order

- - w/o second-order effects
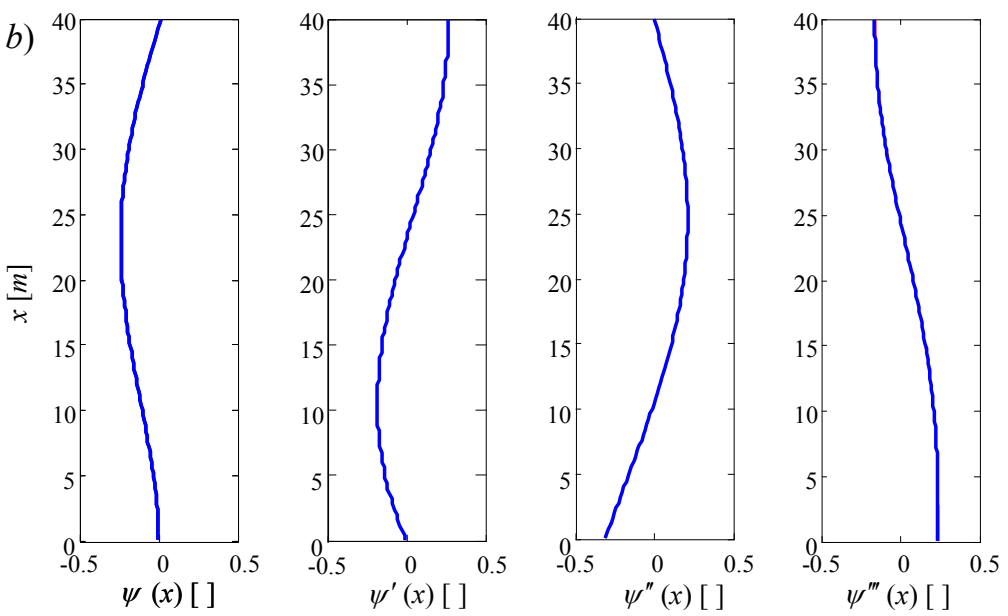

w second-order

- w/o second-order effects

Fig. 3. Comparison of modal shapes and derivatives obtained by accounting for and by disregarding secondorder effects : a) mode 1, b) mode 2 .

\subsection{Seismic response}

In order to evaluate the influence of axial load on the seismic response of the bridge, the pier model has been subjected to a set of 7 real ground motion records [26] compatible to the 
EC8 pseudo-acceleration response spectrum [3]. Fig. 4a reports the acceleration response spectrum of the real records, the mean spectrum, and the code spectrum, whereas Fig. $4 \mathrm{~b}$ reports the displacement response spectrum of the records, and the mean spectrum.

The seismic displacement response to the ground motion record denoted as 000232 ya is reported in Fig. 5, where the time histories of the pier top displacement obtained by accounting for and by disregarding axial load effects are compared. In this figure, the period elongation due to axial load effects can be observed, together with a reduction of the displacement demand of about $15 \%$ with respect to the case disregarding axial load effects. This can be explained by noting that the displacement response is dominated by the fundamental vibration mode, and by observing in Fig. $4 \mathrm{~b}$ that the fundamental period elongation due to axial loads corresponds to a reduction of the spectral displacement ordinates.
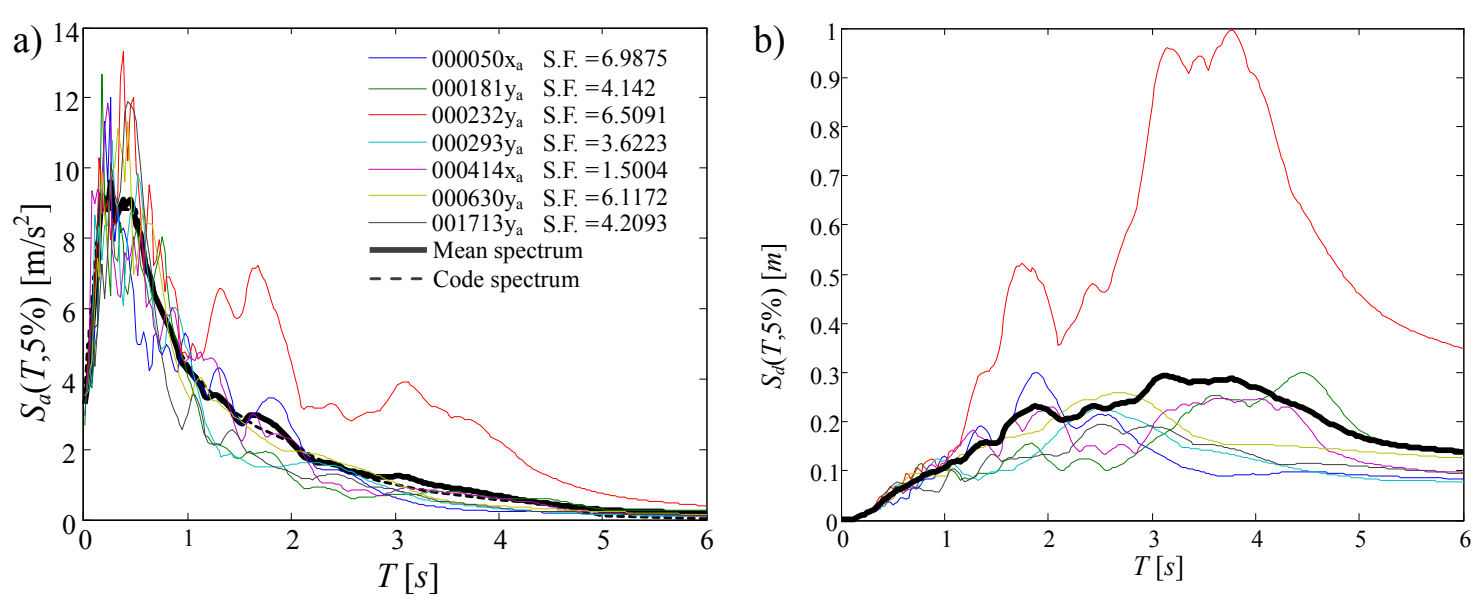

Fig. 4. Acceleration (a) and displacement (b) response spectra of spectrum-compatible natural records.

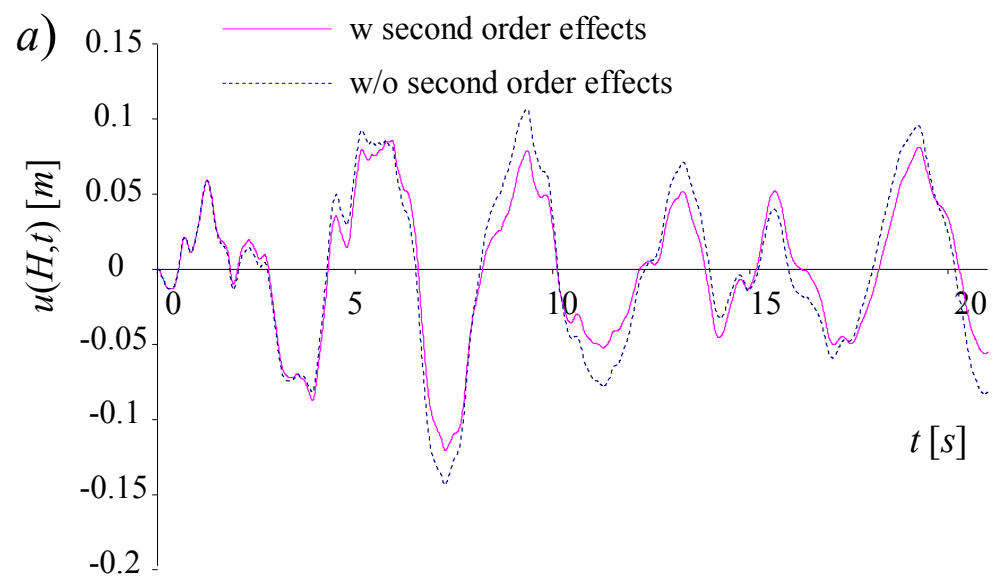

Fig. 5. Time history of pier top displacement under record 000232 ya: comparison of estimate obtained by accounting for and by disregarding axial load effects.

Fig. 6a shows the seismic response envelopes obtained for record 000232ya. The cumulative contributions of the first 6 modes, that account for axial load effects, are plotted separately. It is observed that while the displacement demand is dominated by the first mode contribution only, the shear demand is affected significantly by the higher modes. Also the moment demand at the pier top is slightly influenced by higher modes. However, this effect is not significant for design/assessment purposes, since the moment is highest at the base. 
Fig. $6 \mathrm{~b}$ compares the response envelopes obtained by accounting for and by disregarding axial loads. The increase of vibration period due to axial load effects results in a reduction of the displacement and internal action demands for the pier. The shear demand reduction is less significant than the reduction of the other response parameters. This can be explained by recalling the strong influence of higher vibration modes on the shear demand for this system, and the almost negligible influence of axial load effects on modes of order higher than one.
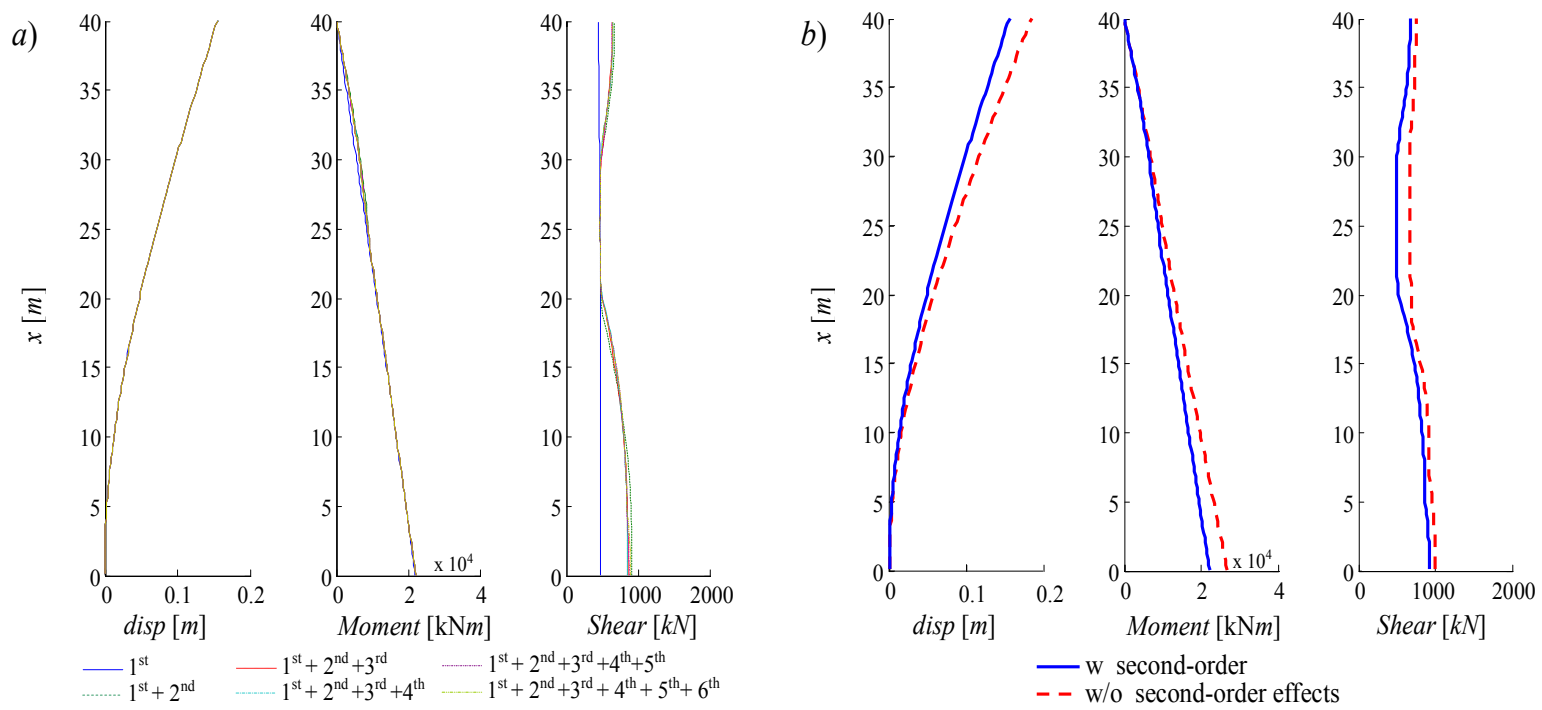

Fig. 6. Seismic response under record 000232 ya: a) Contribution of the first 6 modes to the average seismic response envelopes in terms of displacements, bending moments and shear, b) comparison between the response evaluated by accounting for and disregarding axial load effects.

Fig. 7a shows the average of the peak absolute responses (in terms of displacements, bending moments and shear) evaluated by considering the seven different ground motion records and by accounting for axial load effects. The contribution of the first 6 modes are plotted separately for the response parameters considered. Fig. $7 \mathrm{~b}$ compares the average response envelopes obtained by accounting for and by disregarding axial load effects.
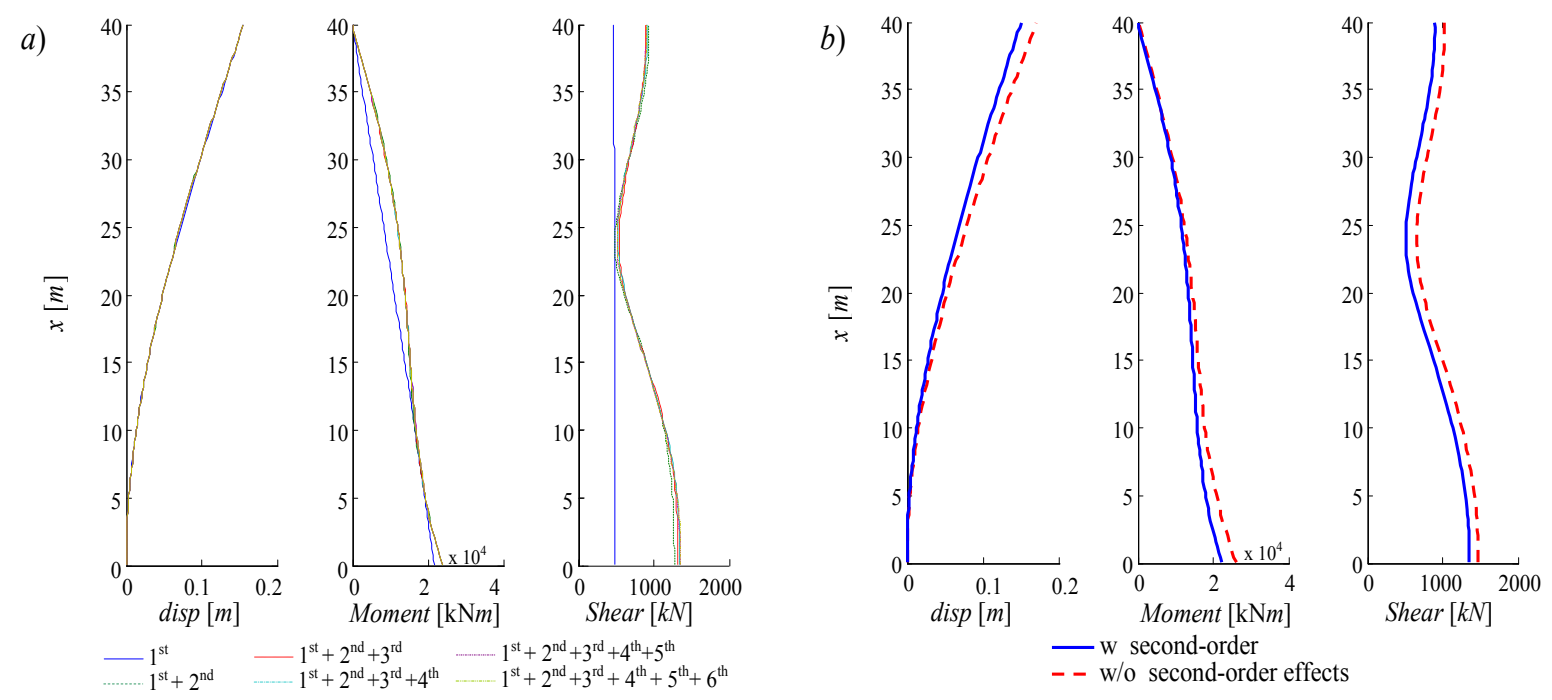

Fig. 7. a) Contribution of the first 6 modes to the average seismic response envelopes in terms of displacements, bending moments and shear, b) comparison between the response evaluated by accounting for and disregarding axial load effects. 
In Fig. 7a, one notes that the displacement demand is dominated by the first mode contribution whereas higher vibration modes contribute more significantly to the shear and bending moment demand. In particular, the first two modes contribute significantly to the bending moments and the first three modes to the shear. Although the bending moment shape is not linear as it would be observed in the case of absence of distributed masses and axial loads, its maximum value is reached at the base section, and is significantly smaller than the yield moment. This confirms that the assumption of linear elastic behaviour for such a slender pier is correct, despite the high level of seismicity of the site. The shear diagram shape also significantly deviates from the constant shape that would correspond to a pier with no distributed mass, thus demonstrating the significant influence of higher vibration modes.

In Fig. $7 b$, it is observed that the axial load effects reduce in general the average response in terms of displacements, moments and shear of about $10 \%$. This result is a consequence of the reduction of the first mode spectral ordinates in terms of both displacements and accelerations (Fig. 4). The observed behaviour contradicts the prescriptions of current seismic design code which use amplification factors for the bending moment demand. In fact, according to EC8-2 [3], the value of the base section bending moment demand evaluated by first order analysis, i.e., by neglecting the axial load effect $\left(M_{E d}\right)$, should be amplified by a factor of 1.1 . On the contrary, the results of this study show that it should be reduced by a factor of 0.946 .

\section{CONCLUSIONS}

In this paper, an analytical technique is presented for investigating the effects of axial loads on the dynamic behaviour and seismic response of slender bridge piers. The technique is based on a continuous model of the pier, which is described by a linear elastic Euler-Bernoulli cantilever beam with uniformly distributed mass, representing the pier mass, and a tip mass at the free end, representing the deck. A concentrated load, acting at the pier top, simulates the pier reaction to deck loads, whereas a distributed axial load simulates the pier self weight. The linearly varying axial load leads to a linear partial differential equation of motion with variable coefficients. This equation is decoupled by using the exact vibration modes of the system, obtained by applying the Frobenius method, and the orthogonality conditions between the modes derived in this study. By this way, the effect of axial loads on the response parameter of interest (i.e., the displacement, the bending moment, and the shear demand along the pier) can be evaluated separately on each vibration mode.

A realistic slender bridge pier is considered as case study to validate the proposed analysis technique and illustrate its capabilities. First, a free-vibration analysis is performed to evaluate the influence of axial load on the pier dynamic behavior. It is observed that axial load effects induce an elongation of the vibration period of the first mode, whereas the higher order modes are less sensitive to these effects. While the displacement shape of the first mode is slightly affected by axial loads, the third order spatial derivative, related to the shear, changes significantly in presence of axial load, and the second order derivative, proportional to the bending moments, changes only moderately. Moreover, the displacement shapes and relevant derivatives of higher modes obtained by accounting for and by disregarding axial load effects are practically indistinguishable.

Successively, the average seismic response to a set of seven compatible natural records in terms of displacement, bending moments and shear envelopes is evaluated and the following conclusions are drawn.

1. The main effect of axial load is to increase the first mode vibration period. The vibration periods, shapes and relevant derivatives of modes of order higher than one are practically not affected by axial loads. 
2. Higher modes notably influence the demand of internal actions and their distribution along the beam.

3. The variation of dynamic properties of the bridge piers due to axial loads may provide significant reduction of the demand in terms of displacements, bending moments, and shear, as observed in this application. By contrast, according to current seismic codes prescriptions, the bending moment seismic demand evaluated by first order analysis should always be amplified to account for axial load effects.

\section{REFERENCES}

[1] D. Bernal, Amplification factors for inelastic dynamic $\mathrm{p}-\Delta$ effects in earthquake analysis. Earthquake Engineering \& Structural Dynamics, 15(5), 635-651, 1987.

[2] L. N. Virgin, R. H. Plaut, Effect of axial load on forced vibrations of beams. Journal of Sound and Vibration, 222, 395-405, 1993.

[3] European Committee for Standardization (ECS), Eurocode 8 - Design of structures for earthquake resistance, Brussels , 2005.

[4] E. Rosenblueth, Slenderness effects in buildings. Journal Structural Division, 91(1), $229-252,1965$.

[5] T. Paulay, A consideration of $\mathrm{p}$ - delta effects in ductile reinforced concrete frames. Bulletin of the New Zealand National Society for Earthquake Engineering, 11(3), 151-160, 1978.

[6] T. Pauley, M. J. N. Priestley, Seismic Design of reinforced concrete and masonry buildings. John Wiley \& Sons INC, 1992.

[7] S. Mahin, R. Boroschek, Influence of geometric nonlinearities on the seismic response and design of bridge structures. Background report to California Department of Transportation, 1991.

[8] R. C. Fenwick, B. J. Davidson, e B. T. Chung, P - delta actions in seismic resistant structures. Bulletin of the New Zealand National Society for Earthquake Engineering, 25(1), 56-69, 1992.

[9] G. A. MacRae, P- $\Delta$ effect on single-degree-of-freedom structures in earthquakes. Earthquake Spectra, 10(3), 539-568, 1994.

[10] M. J. N. Priestley, G. M. Calvi, e M. J. Kowalsky, Displacement-based seismic design of structures. IUSS Press, 2007.

[11] B. Wei, Y. Xu, e J. Li, Treatment of P- $\Delta$ Effects in Displacement-Based Seismic Design for SDOF Systems. Journal of Bridge Engineering, 17(3), 509-518, 2012.

[12] M. Aschheim, E. H. Montes, The representation of P- $\Delta$ effects using Yield Point Spectra. Engineering Structures, 25, 1387-1396, 2003.

[13] K. Chopra, Dynamics of structures: Theory and Applications to Earthquake Engineering. Prentice - Hall Inc., 1995.

[14] Z. Guan, J. LI, Y. XU, H. LU, Higher-order mode effects on the seismic performance of tall piers. Frontiers of Architecture and Civil Engineering in China, 5(4), 496-502, 2011. 
[15] R. Ceravolo, G.V. Demarie, L. Giordano, G. Mancini, D. Sabia, Problems in applying code-specified capacity design procedures to seismic design of tall piers. Engineering Structures, 31(8), 1811-1821, 2009.

[16] R. P. Dhakal K. Maekawa, Analytical Prediction of Collapse of RC Piers Induced by Geometrical Nonlinearity. The First International Conference on Structural Stability and Dynamics, Taipei, Taiwan, 2000.

[17] H.-G. Kwak, J.-K. Kim, Effect of slender RC columns under seismic load. Engineering Structures, 29(11), 3121-3133, 2007.

[18] A. Bokaian, Natural frequencies of beams under compressive axial loads. Journal of Sound and Vibration, 126(1), 49-65, 1988.

[19] S. Naguleswaran, Transverse vibration of an uniform Euler-Bernoulli beam under linearly varying axial force. Journal of Sound and Vibration, 275(1-2), 47-57, 2004.

[20] I. Elishakoff, EIGENVALUES OF INHOMOGENEOUS STRUCTURES Unusual Closed-Form Solutions. CRC PRESS, 2005.

[21] S. S. Rao, Vibration of Continuous Systems. Wiley, New York, 2007.

[22] A. Dall'Asta, G. Leoni, Vibrations of beams prestressed by internal frictionless cables. Journal of Sound and Vibration, 222(1), 1-18.

[23] E. Tubaldi, A. Dall'Asta. Transverse free vibrations of continuous bridges with abutment restraint. Earthquake Engineering \& Structural Dynamics, 41(9), 1319-1340, 2012.

[24] Y. Bouassida, E. Bouchon, B. Kolias et al., Bridge Design to Eurocodes Worked examples. A. Athanasopoulou, M. Poljansek, A. Pinto, G. Tsionis, S. Denton eds. Workshop "Bridge Design to Eurocodes", Vienna, Austria, October 4-6, 2010.

[25] European Committee for Standardization (ECS), Eurocode 2 - Design of concrete structures, Brussels , 2004.

[26] Iervolino, C. Galasso, E. Cosenza, REXEL: computer aided record selection for codebased seismic structural analysis. Bulletin of Earthquake Engineering, 8(2), 339-362, 2009.

\section{APPENDIX A: EIGENVALUE PROBLEM SOLUTION BY THE FROBENIUS METHOD}

The eigenvalue problem is solved for the case with uniform distributed mass and stiffness along the spatial variable. Substituting $b(x)$ by $E I$ and $m(x)$ by $\bar{m}$ into Eqn. (7) the strong form of the seismic problem can be conveniently rewritten as

$$
\left\{\begin{array}{l}
\psi^{i v}(x)+g \psi^{\prime \prime}(x)-h\left[x \psi^{\prime}(x)\right]^{\prime}+\Omega^{4} \psi(x)=0 \\
\psi(0)=\psi^{\prime}(0)=\psi^{\prime \prime}(H)=0 \\
E I \psi^{\prime \prime \prime}(H)+P \psi^{\prime}(H)+\omega^{2} M \psi(H)=0
\end{array}\right.
$$

where $g=\left(\frac{P}{E I}+\frac{\bar{m} g H}{E I}\right), h=\frac{\bar{m} g}{E I}$, and $\Omega^{4}=-\frac{\bar{m}}{E I} \omega^{2}$. 
The Frobenius method seeks the solution to Eqn.(A1) in the form

$$
\psi(x, c)=\sum_{n=0}^{n=\infty} a_{n+1}(c) x^{c+n}
$$

in which the coefficients $a_{n+1}$ are functions of the undetermined exponent $c$.

By differentiating Eqn. (A2), and substituting into Eqn.(A1), one has

$$
\begin{aligned}
& \sum_{n=0}^{n=\infty} a_{n+1}(c)(c+n)(c+n-1)(c+n-2)(c+n-3) x^{c+n-4}+ \\
& g a_{n+1}(c)(c+n)(c+n-1) x^{c+n-2}-h a_{n+1}(c)(c+n)^{2} x^{c+n-1}+\Omega^{4} a_{n+1}(c) x^{c+n}=0
\end{aligned}
$$

From Eqn. (A3) one obtains an indicial equation for $c$ and a set of equations for the coefficients $a_{n+1}$ as shown below.

Without loss of generality, the lead coefficient $a_{1}$ is assumed equal to 1 . The allowable values for $c$ are the roots of the indicial equation, obtained by setting the lowest power of $x$ in Eqn.(A3) (i.e., $c$-4) equal to zero

$$
a_{1}(c) c(c-1)(c-2)(c-3)=0
$$

Based on Eqn. (A4), Eqn. (A3) is rewritten as

$$
\begin{aligned}
& \sum_{n=0}^{n=\infty} a_{n+2}(c)(c+n+1)(c+n)(c+n-1)(c+n-2) x^{c+n-3}+ \\
& g a_{n+1}(c)(c+n)(c+n-1) x^{c+n-2}-h a_{n+1}(c)(c+n)^{2} x^{c+n-1}+\Omega^{4} a_{n+1}(c) x^{c+n}=0
\end{aligned}
$$

The summations indices of the four terms of the sum are then shifted to obtain a common power of $x$ equal to $(c+n-3)$.

$$
\begin{aligned}
& \sum_{n=0}^{n=\infty} a_{n+2}(c)(c+n+1)(c+n)(c+n-1)(c+n-2) x^{c+n-3}+\sum_{n=3}^{n=\infty} \Omega^{4} a_{n-2}(c) x^{c+n-3}+ \\
& \sum_{n=1}^{n=\infty} g a_{n}(c)(c+n-1)(c+n-2) x^{c+n-3}+\sum_{n=2}^{n=\infty}-h a_{n-1}(c)(c+n-2)^{2} x^{c+n-3}=0
\end{aligned}
$$

From Eqn. (A6) to be zero, each power of $x$ must vanish and, thus:

$$
\begin{aligned}
& a_{n+2}(c)(c+n+1)(c+n)(c+n-1)(c+n-2)+g a_{n}(c)(c+n-1)(c+n-2) \\
& -h a_{n-1}(c)(c+n-2)^{2}+\Omega^{4} a_{n-2}(c)=0
\end{aligned}
$$

for all the allowed $n$. This gives the following recurrence relationship:

$$
a_{n+2}(c)=-\frac{g a_{n}(c)(c+n-1)(c+n-2)-h a_{n-1}(c)(c+n-2)^{2}+\Omega^{4} a_{n-2}(c)}{(c+n+1)(c+n)(c+n-1)(c+n-2)}
$$

subject to $a_{k}(c)=0$ for $k \leq 0$.

For $c=0,1,2,3$ and $n=0,1,2$, Eqn.(A8) gives: 


$$
\begin{aligned}
& a_{2}(0)=a_{2}(1)=a_{2}(2)=a_{2}(3)=0 \\
& a_{3}(0)=-\frac{g}{2}, a_{3}(1)=-\frac{g}{6}, a_{3}(2)=-\frac{g}{12}, a_{3}(3)=-\frac{g}{20} \\
& a_{4}(0)=0, a_{4}(1)=\frac{h}{24}, a_{4}(2)=\frac{h}{30}, a_{4}(3)=\frac{h}{40}
\end{aligned}
$$

The four power series solutions that satisfy Eqn.(A1), each corresponding to a root of Eqn.(A4), are:

$$
\begin{aligned}
& \psi(x, 0)=\psi_{0}(x)=\sum_{n=0}^{n=\infty} a_{n+1}(0) x^{n} \\
& \psi(x, 1)=\psi_{1}(x)=\sum_{n=0}^{n=\infty} a_{n+1}(1) x^{n+1} \\
& \psi(x, 2)=\psi_{2}(x)=\sum_{n=0}^{n=\infty} a_{n+1}(2) x^{n+2} \\
& \psi(x, 3)=\psi_{3}(x)=\sum_{n=0}^{n=\infty} a_{n+1}(3) x^{n+3}
\end{aligned}
$$

The general modal shape $\psi(x)$ is obtained by combination of these four power series linearly independent:

$$
\psi(x)=C_{0} \psi_{0}(x)+C_{1} \psi_{1}(x)+C_{2} \psi_{2}(x)+C_{3} \psi_{3}(x)
$$

Substituting Eqn. (A11) into the boundary conditions at the clamped end one obtains $C_{0}=C_{1}=0$ whereas the boundary conditions at the free end give:

$$
\left\{\begin{array}{l}
C_{2} \psi_{2}^{\prime \prime}(H)+C_{3} \psi_{3}^{\prime \prime}(H)=0 \\
C_{2}\left[E I \psi_{2}^{\prime \prime \prime}(H)+P \psi_{2}^{\prime}(H)+\omega^{2} M \psi_{2}(H)\right]+ \\
C_{3}\left[E I \psi_{3}^{\prime \prime \prime}(H)+P \psi_{3}^{\prime}(H)+\omega^{2} M \psi_{3}(H)\right]=0
\end{array}\right.
$$

Eqn. (A12) corresponds to a homogeneous system of two equations in the unknown $C_{2}$ and $C_{3}$. In order to obtain a non-trivial solution, the determinant of the coefficient matrix must to be equal to zero, thus yielding the following transcendental equation in the eigenvalue $\lambda$ :

$$
\begin{aligned}
& \psi_{2}{ }^{\prime}(H)\left(E I \psi_{3}{ }^{\prime \prime}(H)+P \psi_{3}{ }^{\prime}(H)+\omega^{2} M \psi_{3}(H)\right)- \\
& -\psi_{3}{ }^{\prime \prime}(H)\left(E I \psi_{2}{ }^{\prime \prime}(H)+P \psi_{2}{ }^{\prime}(H)+\omega^{2} M \psi_{2}(H)\right)=0
\end{aligned}
$$

This equation yields an infinite set of eigenvalues. The corresponding eigenvectors are obtained from Eqn.(A12), for which $C_{3}=-C_{2} \psi_{2}{ }^{\prime \prime}(H) / \psi_{3}{ }^{\prime \prime}(H)$, and their expression is:

$$
\psi(x)=C_{2}\left[\psi_{2}(x)-\frac{\psi_{2}^{\prime \prime}(H)}{\psi_{3}^{\prime \prime}(H)} \psi_{3}(x)\right]
$$

